\title{
LA CONSTITUCIÓN DE ACTORES DE LA CALIDAD EN EDUCACIÓN SUPERIOR DESDE EL INSTITUCIONALISMO CENTRADO EN ACTORES EL CASO DE AQU CATALUÑA Y SU EVOLUCIÓN DESDE CONSORCIO A AGENCIA
}

\section{Introducción}

En el marco del procesamiento de las políticas públicas, en oportunidades el tratamiento técnico de los problemas sustantivos que explican la promoción de diversas iniciativas públicas posterga la reflexión acerca de los procesos que originan la construcción, promoción y concreción de ellas. Sobre el particular, la perspectiva del Actor-Centered Institutionalism ${ }^{1}$ (ACI) propuesta por Scharpf (1997) reconoce dos posibles orientaciones de la investigación en materia de políticas públicas: una referida al problema y otra dirigida a la interacción. Mientras la primera pone su foco en aquellos aspectos más sustantivos de la política (¿qué pasa?, ¿cuál es el problema? y en consecuencia ¿qué hacer?); la segunda -la que aquí privilegiaremosfija su atención en aquellos aspectos de la política que derivan de la interacción entre diversos actores para la conceptualización, la formulación y la implementación de las correspondientes políticas.

No obstante, para algunas recientes y valiosas iniciativas en la materia (Geoffroy, 2013) en el ámbito de las políticas de promoción y aseguramiento de la calidad un tratamiento como el antes propuesto es algo raro, en tanto lo usual es que el diálogo en torno a las políticas de calidad ponga su atención en las temáticas y problemáticas técnicas, más que en los procesos que estas enfrentan para ser implementadas. Lo anterior resulta comprensible si pensamos que, en ciertas oportunidades, el tratamiento de los elementos sustantivos de las políticas públicas se encuentra hegemonizado por aproximaciones tecnocráticas, lo que de alguna

Institucionalismo Centrado en Actores. 
forma encubre y naturaliza la construcción procesual y contextual de dichas iniciativas.

De manera distinta, lo que una perspectiva basada en lo interactivo de la implementación pretende poner de manifiesto, tiene que ver con la contingencia a la cual las políticas públicas se enfrentan al momento de ser ejecutadas. Dicha contingencia se relaciona con una dimensión tal vez menos conceptualizada, siendo no obstante una de las que mayor fuerza le brinda en el ámbito de lo operativo, en tanto se vincula con la forma mediante la cual la política pública se concreta en el espacio público, configurando y enfrentando a actores con diversas agendas de intereses.

Valga esto también para el caso de las políticas de promoción y aseguramiento de la calidad en la educación superior, que releva la importancia de prestar atención a los procesos de construcción de política, muchas veces omitidos. En lo efectivo, podríamos pensar que en la implementación de un sistema de aseguramiento de la calidad de programas no es indiferente si este sistema deposita la responsabilidad de su coordinación en una serie de agencias parciales, o en una agencia única; de ser este último el caso, tampoco es indiferente si esta agencia es estatal, proveniente de las propias universidades, o bien conformada como una instancia mixta. En lo efectivo, nunca resulta algo inocuo que la configuración de roles de una agencia y la forma en que esta se vincula con las universidades oscile entre una instancia donde las universidades son directamente parte de ella, u otra en la que su alto nivel de autonomía la distancia de las organizaciones universitarias.

Así, el presente trabajo pretende, a partir de la revisión del caso de la creación, constitución y posterior evolución de la agencia catalana para el aseguramiento de la calidad, poner de manifiesto la forma en que las modificaciones en la constitución de los actores traen asociadas cambios en la manera en que estos son percibidos y, en definitiva, cómo interactúan con actores relevantes en los distintos momentos del procesamiento de las políticas de calidad, al interior de algunas universidades catalanas. Previo a eso se aborda el proceso de configuración de la mencionada agencia, en tanto actor prioritario de las políticas de calidad en la educación superior, considerando que 
durante las últimas décadas la historia de las políticas de promoción y aseguramiento de la calidad en la comunidad autónoma de Cataluña resulta difícilmente separable de lo que ha sido la historia de la constitución y desarrollo de esta.

\section{Institucionalismo Centrado en Actores y las políticas públicas}

Cuando hablamos del ACI no hablamos de una teoría compleja ni acabada, sino de un marco conceptual que nos permitirá aportar elementos que ayuden a entender la forma en que los temas de la política pública son procesados a través de la interacción entre actores (individuales, colectivos u organizados) dotados de ciertas capacidades y orientaciones (regulativas, cognitivas y normativas), provistas por el contexto institucional al interior del cual se desenvuelven. Específicamente, el ACI -desarrollado por Renate Mayntz y Fritz Scharpf- (Scharpf, 1989, 1997, 2000 a, 2000 b; Zurbriggen, 2006; Navarro Yánez, 2002) sostiene que cada problema que es objeto de atención por parte de las políticas públicas tiene lugar en un contexto institucional que da cuenta de los actores que participan en los procesos de interacción, sus orientaciones y capacidades, pudiendo derivar de ello posibles cursos o modos de interacción, así como resultados específicos de las políticas que se traten (Scharpf, 1997). En lo específico, y tras la adopción de las correspondientes políticas públicas, aquello que resulte y pueda ser implementado es producto del procesamiento que hagan de ello diversos actores que interactúan entre sí (ver Esquema 1). 
Esquema 1: El ámbito de la investigación orientadas a la interacción en las políticas públicas. Fuente: extraído de Scharpf (1989).

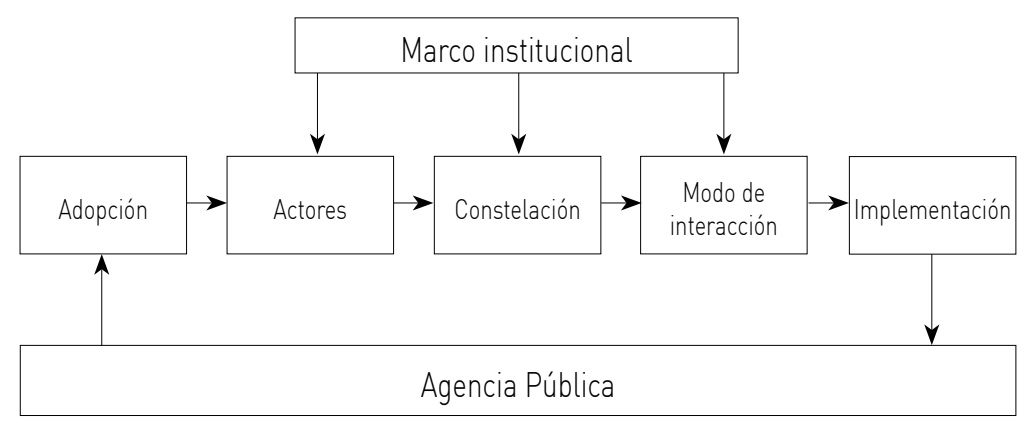

Como perspectiva interactiva, el ACI destaca la complejidad de la implementación de las políticas públicas, enfocándose en las relaciones de intercambio entre diversos actores (Enders, Jeliazkova, McGuiness \& Massen, 2003). En dichas interacciones pueden manifestarse tanto actores individuales como colectivos, todos ellos dotados de la capacidad de desarrollar acciones provistas de diferentes propósitos; no obstante se puede observar que el ACI presta especial atención a los actores corporativos u organizaciones.

En este mismo marco institucional puede observarse la existencia de distintas orientaciones y modos de interacción (acciones unilaterales, negociaciones, interacciones instructivas, etc.) por medio de los cuales los actores, interactuando e influenciándose mutuamente, dan forma a los resultados de las respectivas políticas públicas.

Basado en una perspectiva neoinstitucional y utilizando una lógica situacional emparentada con la teoría de juegos, el ACI supone que cada política pública configura un sistema interactivo, que pone recursos a disposición de los actores, así como una gama más o menos amplia de posibles modos de interacción o, si se prefiere, una estructura de oportunidades específica. A ello añade que determinados marcos institucionales (institutional settings) hacen más o menos probable el desarrollo de modos de interacción específicos (Scharpf, 1997). Desde esta perspectiva, el contexto institucional sería 
un elemento fundamental para configurar y así explicar los modos de interacción que tienen lugar entre los actores, en el marco de la implementación de una política pública (Navarro Yánez, 2002).

En opinión de Zurbriggen (2006), de esta forma los actores públicos y privados no son inherentemente libres, en tanto enfrentan una serie de posibilidades y restricciones provenientes del marco político-institucional más o menos permanente en el espacio y el tiempo. Sin embargo, la creación institucional es un proceso continuo, sustentado sobre la base de reglas construidas de manera social en determinados contextos históricos y políticos. No obstante, por muy sólida y permanente que pueda parecer una institución, los agentes pueden elegir determinadas opciones y romper ciertas reglas. De esta forma, estas no son permanentes y pueden cambiar a partir del accionar y de las decisiones adoptadas por los agentes dentro del marco institucional respectivo, siendo motivadas tanto por factores internos como externos. Lo importante en este punto es entender lo improbable que es que cualquier actor-concebido como una entidad unificada capaz de acción- sea capaz de determinar por sí solo el resultado de las políticas, según sus propias percepciones, preferencias y capacidades. Lo que es determinante es la forma mediante la que se configura el sistema interactivo de todos los actores involucrados en la correspondiente política, considerando sus propias percepciones, preferencias y capacidades. La forma en la que queda configurado este sistema de interacciones es aquello que Scharpf denomina "constelación de actores".

Así visto, lo que resulta de las políticas (en el caso que nos atañe, del proceso de implementación de estas) dependerá de la forma en que los diversos actores, a partir de sus percepciones, capacidades y preferencias, se constituyan e interactúen entre sí. Vale decir, de la manera en la cual los actores puedan jugar y jueguen efectivamente el juego. Según Navarro Yánez (2002), desde esta perspectiva el ACI facilita el desarrollo de modelos analíticos que permiten comparar la forma en que se desarrollan los procesos propios de las políticas públicas (entre otros la conceptualización, el diseño y la implementación) en distintos contextos interactivos e institucionales. 
Valga lo anterior para señalar que, en oportunidades, en el ámbito de las políticas públicas, surge la necesidad de privilegiar una aproximación más cercana a lo que Scharpf señala como Interaction Oriented Policy Research (Investigación Orientada a la Interacción en las Políticas Públicas), por sobre la aproximación más sustantiva (y a veces esencialista, como en el caso de las políticas de aseguramiento de la calidad) orientada a la resolución de problemas. Después de todo, aquello en lo que consiste el problema respectivo no es algo en sí mismo; sino más bien el constructo que resulta de un proceso interactivo. Esto cobra especial relevancia en el caso de las políticas que promueven la calidad en el ámbito de la educación superior, concepto respecto del cual algunos autores señalan que no sería unívoco y fijo, sino que, más allá de su polisemia (Poole, 2005; Harvey $\&$ Green, 1993), se construye por consensos y negociaciones entre los actores (Fernández Lamarra, 2007), llegando algunos autores incluso a sugerir que el tema de la calidad sea abordado como un discurso ideológico (Lemaitre, 2002), o bien, un campo de posiciones políticas (Perellón, 2005).

Así visto, un análisis más orientado a la interacción entre agentes permitiría abordar, entre otros aspectos, la negociación de estos significados a partir de actores diversos en distintas posiciones y disposiciones a la colaboración, competición o al conflicto; pudiendo revelarnos de mejor manera en qué instancias del marco institucional radican los problemas dotados de una grado mayor de inteligibilidad para su adecuado tratamiento; las más de las veces mejor dispuestas para el tratamiento del problema que algunos discursos en torno a la calidad.

En este sentido, el procesamiento de las políticas es referido como un campo de juego de múltiples actores que tiende a incorporar partes interesadas que regularmente redefinen el rol y el equilibrio de poder entre aquellas tradicionalmente implicadas (Enders et al., 2003).

Para ilustrar lo antes señalado, a continuación se revisará el caso de la creación, constitución y posterior evolución de la agencia catalana para temas de calidad en la educación universitaria, intentando definir 
cómo el cambio desde la noción original de un consorcio participativo al de una agencia autónoma en plenitud tensiona y modifica la manera en que el accionar de la agencia es percibida por actores internos a las universidades, donde sus iniciativas de aseguramiento de la calidad son implementadas.

La narración del caso está basada en una revisión documental (principalmente al momento de recrear la historia y la génesis de la agencia respectiva) como también en entrevistas realizadas tanto a funcionarios de la agencia como a autoridades al interior de las universidades. En el caso de las entrevistas y, en el marco de los compromisos propios de la investigación, se ha desarrollado una estrategia de anonimatización.

\section{AQU Cataluña: breve historia y contexto preliminar}

Durante la década de los noventa, la necesidad de garantizar la calidad en la educación superior irrumpe con fuerza en diversos países como Francia, los Países Bajos o el Reino Unido. España no está ajena a esta tendencia, adhiriendo al conjunto de países que se deja seducir por la perspectiva de la calidad en la educación superior y la necesidad de apostar por ella.

A comienzos de esa misma década, y siguiendo la corriente europea en esta materia, algunas universidades de la comunidad autónoma de Cataluña -principalmente aquellas situadas en su capital, Barcelona-iniciarán, de manera experimental, diversas iniciativas en materia de evaluación de la calidad en algunas de sus titulaciones. Fue así como, promediando el decenio, Cataluña parecía ir a la delantera del Estado español en materia de políticas de calidad en la educación superior, mostrando gran implicación en el desarrollo de estas, tanto por parte de sus universidades como de la Generalitat ${ }^{2}$. Esta situación favoreció que el tema de la calidad se constituyese como un espacio de encuentro para el fortalecimiento del sistema universitario catalán ${ }^{3}$.

2 Institucionalidad propia que se da para efectos de su autogobierno la comunidad autónoma de Cataluña en España.

3 Publicación AQU: L'impacte de les avaluacions a les universitats catalanes (1996 - 2002). 
En este sentido, las primeras experiencias posibilitarán que, posteriormente, algunas universidades catalanas participaran en un programa experimental de evaluación de la calidad del sistema universitario, llevado a efecto a nivel nacional por el Estado español. Dicho programa fue promovido por el Consejo de Universidades en 1992 y desarrollado durante 1993 y 1994. De la misma forma fue promocionada la participación de una universidad catalana en el proyecto piloto europeo de calidad en la educación superior ${ }^{4}$.

Estos proyectos experimentales tenían como principal objetivo ensayar y establecer metodologías adecuadas para la evaluación de las titulaciones universitarias en el marco europeo. Sin embargo, no fue sino hasta el bienio 1996-1997 que la evaluación de la calidad se generaliza en las universidades catalanas ${ }^{5}$.

La generalización de estas prácticas se debió, en primer lugar, a la puesta en marcha por parte del gobierno central español, por medio del Ministerio de Educación y Ciencia, del Plan Nacional de Evaluación de la Calidad de las Universidades (PNECU); y en segundo, a la creación por parte de las universidades públicas catalanas y de la Generalitat de Cataluña, a fines de 1996, de l'Agència per a la Qualitat del Sistema Universitari a Cataluny ${ }^{6}$.

A la orden ministerial del 21 de febrero de 1996 que abre la primera convocatoria del PNECU, le sigue en el ámbito de la comunidad autónoma de Cataluña la resolución de la presidencia de la Generalitat del 20 de marzo de 1996, por medio de la que se convoca a las universidades catalanas a concursar por financiamiento para el desarrollo de proyectos de evaluación de la calidad ${ }^{7}$.

A pesar de que el PNECU tenía una inspiración bastante centralista impulsada por el gobierno de Madrid, contemplaba la posibilidad de incorporar algunas propuestas que, en materia de

AQU Cataluña proceso de evaluación de la calidad del sistema universitario a Cataluña, Informe 1999.

Ver referencia en pie de página $\mathrm{n}^{\circ} 3$.

Ver referencia en pie de página $\mathrm{n}^{\circ} 3$.

Publicación AQU:L'Avaluació de la Qualitat Universitària. Taller de Girona, setembre de 1998 15 al 17 de setembre de 1998 a la Universitat de Girona. 
calidad, presentasen las comunidades autónomas, a las que ya habían sido traspasadas las funciones en materia universitaria ${ }^{8}$, aun cuando algunas de ellas, como la Generalitat, no las habían terminado de asumir 9 . En este contexto, el PNECU sería desarrollado por medio de una fórmula cooperativa entre el Consejo de Universidades (organismo centralizado del gobierno español), que promocionaba el plan, y las comunidades autónomas que, como Cataluña, decidieron administrar su participación en este de forma directa, una vez que fuera implementado ${ }^{10}$. Como señala un funcionario de la agencia: (...) el 97 y el 96 en concreto, ya se visualizó la necesidad de disponer en Cataluña de un instrumento que promoviera todas estas politicas de calidad en Cataluña ${ }^{11}$.

Los responsables de las universidades catalanas, la administración y las mismas universidades se hacen conscientes de la necesidad de crear una agencia para la calidad del sistema universitario, en proporción a los modelos que, en ese entonces, desarrollaban en Europa los países preocupados por mejorar la calidad del servicio público ofrecido por las universidades, respetando la autonomía de estos centros ${ }^{12}$.

En este contexto, con el reto de mejorar la calidad de los servicios universitarios y con la clara visión de que este ámbito resultaría claro en un futuro más inmediato la Generalitat de Catalunya va a promover la creación de una agencia que tuviese como objetivo la mejora de la calidad del sistema universitario catalán ${ }^{13}$.

No obstante lo anterior, había ciertas aprensiones respecto de la naturaleza de la nueva entidad, pues existía el peligro de crear un organismo más controlador que impulsor de los sistemas de calidad en las universidades, ya que ni en el mundo universitario ni en la

8 Ver referencia en pie de página $\mathrm{n}^{\circ} 3$.

9 A pesar de que la Generalitat tenía competencia plena en el ámbito universitario, muchos aspectos relacionados con las titulaciones hacían referencia al gobierno central.

10 Publicación AQU: Taller ENQA Ministerio de Educación Cultura y Deporte.

11 Entrevista a personal técnico de AQU.

12 Publicación AQU: La integració de les universitats a l'espai europeu. Taller de Girona, 10 i 11 de gener de 2002.

13 Ver referencia en pie de página $\mathrm{n}^{\circ} 3$. 
administración pública existía la tradición de evaluar la calidad institucional ${ }^{14}$.

Es así como, con el reto de mejorar la calidad universitaria, el 26 de septiembre de 1996 las universidades públicas catalanas, representadas por sus rectores, y la Generalitat de Cataluña, representada por el Comissionat dUniversitats i Recerca, acuerdan constituir de manera conjunta, bajo la figura jurídica de un consorcio, l'Agència per a la Qualitat del Sistema Universitari a Catalunya ${ }^{15}$.

La creación de la agencia se lleva a cabo en un entorno donde el marco jurídico aplicable en el sistema universitario catalán no trata específicamente del tema de la garantía de la calidad, ni el uso de la evaluación como un sistema de rendición de cuentas a la sociedad. Solo posteriormente con la Ley Orgánica de Universidades (LOU, 2001) y en lo que respecta a la realidad catalana con la Ley de Universidades de Cataluña (LUC, 2003) se hará presente que la sociedad tiene derecho a exigir calidad docente e investigadora, indicando que la rendición de cuentas ha de ser la debida contrapartida de la autonomía y financiamiento de las universidades ${ }^{16}$.

El 4 de diciembre de 1996 la resolución del Comissionat per a Universitats i Recerca concreta el programa de las evaluaciones institucionales de las universidades públicas y privadas de Cataluña ${ }^{17}$. En este contexto, el mismo Comissionat le encomienda la gestión del programa de evaluación institucional que se está iniciando a la recién creada Agència per a la Qualitat del Sistema Universitari a Catalunya ${ }^{18}$.

En este contexto, el modelo de evaluación a aplicar por la Generalitat deriva del consensuado en su momento por la Unión Europea, en el marco del plan piloto en esta materia, circunstancia en la cual es homologable a los modelos empleados por los países del

\footnotetext{
14 Ver referencia en pie de página $n^{\circ} 12$.

15 AQU Cataluña, proceso de evaluación de la calidad del sistema universitario a Cataluña, Informe 1998.

16 Ver referencia en pie de página $\mathrm{n}^{\circ} 3$.

17 Ver referencia en pie de página $n^{\circ} 15$

18 Ibíd.
} 
correspondiente entorno continental ${ }^{19}$. Con este fin la agencia recibió subvenciones para transferirlas a las universidades participantes con las que firmó convenios en los que se establecía, entre otras cosas, el programa de evaluaciones de cada una, la metodología y los plazos previstos ${ }^{20}$. Además de representar a las universidades en la solicitud de subvenciones, la agencia recibió de parte de la Generalitat el financiamiento necesario para el desarrollo de las acciones necesarias de apoyo a las evaluaciones, las que constituyeron su programa inicial de actividades ${ }^{21}$.

En términos generales, el PNECU establecía dos modalidades de participación ${ }^{22}$ : una basada en disponer de un proyecto global de evaluación, de forma tal que en un plazo de cinco años se hubiesen evaluado todos los programas de pregrado de una universidad; y otra consistente en proyectos temáticos que establecían la evaluación parcial de los mismos ${ }^{23}$. Frente a esto, todas las universidades públicas catalanas se presentaron voluntariamente a la convocatoria de la Generalitat del 20 de marzo de 1996, no obstante el hecho de que las propuestas realizadas tenían distinto alcance según cada universidad ${ }^{24}$.

Una vez acabado el proceso comprendido en la primera convocatoria se va a considerar oportuno analizar y reflexionar sobre la metodología empleada en la evaluación, los indicadores utilizados, la respuesta que esta experiencia había tenido en las unidades evaluadas, etc. Con el objeto de detectar los puntos sólidos y débiles de este primer proceso y proponer las mejoras que convendría introducir en las etapas posteriores $^{25}$.

Al iniciarse la segunda parte del PNECU, pese a que en opinión de los directivos de AQU en la gestión de este programa se había contado con plena autonomía, desarrollando en general una buena

19 Ibíd.

20 Ver referencia en pie de página $\mathrm{n}^{\circ} 4$.

21 Ver referencia en pie de página $n^{\circ} 7$.

22 Además de algunas instancias específicas como las desarrolladas, en el caso de Cataluña, por universidades privadas.

23 El modelo catalán de agencia para la garantía de la calidad universitaria; Directora Cuadernos para la Educación Superior (Ex-IRC). Acceso WEB 01-10-1999.

24 Ver referencia en pie de página $\mathrm{n}^{\circ} 15$.

25 Ver referencia en pie de página $\mathrm{n}^{\circ} 7$. 
relación con el Consejo de Universidades (órgano centralizado español para las universidades ${ }^{26}$, la comunidad autónoma de Cataluña decidió no seguir participando del proceso, y con la experiencia acumulada optó por iniciar un camino propio en materia de la evaluación de la calidad de sus universidades ${ }^{27}$.

\section{Génesis de AQU Cataluña como consorcio}

Las universidades catalanas se configuran como un sistema a partir de la aprobación en 1978 de la Constitución Española, donde prevé que las Comunidades Autónomas puedan asumir competencias en materia de gestión universitaria; y, por otra, de la aprobación en 1979 del Estatuto de Autonomía de Cataluña, el que establece que es competencia plena del Gobierno de la Generalitat de Cataluña la regulación y la administración de la enseñanza, en toda su extensión, modalidades, niveles y grados. Posteriormente, la transferencia explícita de la competencia de coordinación de las universidades de Cataluña a la Generalitat ${ }^{28}$ posibilitó, entre otras cosas, el establecimiento de nuevas políticas universitarias (López Pereira, 2004) ${ }^{29} 30$. En este sentido, probablemente uno de los momentos más importantes en el desarrollo de este tipo de políticas fue la creación del consorcio Agència per a la Qualitat del Sistema Universitari a Cataluña (AQU Cataluña) ${ }^{31}$ 32, en octubre de 1996.

La evolución de la enseñanza universitaria en los últimos años, tanto en Cataluña como en los países de nuestro entorno, nos indica que ha llegado el momento de poner en primer término la

26 Ver referencia en pie de página $\mathrm{n}^{\circ} 12$.

27 Contextualización Histórica AQU. Esquema formalizado dibujado por alto asesor.

28 Artículo No 3, Punto 3 Ley de Reforma Universitaria (LRU) 1983; Ley de Coordinación Universitaria y de Creación de Consejos Sociales (ley de diciembre de 1984 de la C. A. de Cataluña publicada por el BOE nº19 de 22/1/1985).

29 Según el autor, las dos principales competencias autonómicas consistirían en la obligación de financiar las actividades docentes y de investigación en las universidades, y la de exigir calidad en el desempeño de estas actividades a las organizaciones universitarias.

30 Ver referencia en pie de página $\mathrm{n}^{\circ} 23$.

31 Extracto Boletines AQU.

32 Al respecto, cabe señalar que al momento de crearse la agencia el sistema universitario catalán estaba compuesto por nueve universidades: siete públicas y dos privadas. El sistema agregado arrojaba 171.061 estudiantes y 12.244 profesores que impartían 150 programas de estudio diferentes en más de 400 centros (ver referencia en pie de página no 3 ). 
preocupación por la calidad universitaria. Las universidades públicas catalanas y el Comissionat per Universitats i Recerca van a entender eso al crear la Agència per a la Qualitat del Sistema Universitari a Catalunya como un consorcio con personalidad jurídica propia e independiente de sus miembros ${ }^{33}$.

Es así como por medio del Decreto No 355/1996 (emitido por la Presidencia de la Generalitat el 29 de octubre y publicado en el Diari Oficial de la Generalitat de Cataluña el 6 de noviembre de 1996) se constituyó, bajo la figura jurídica de consorcio, la Agència per a la Qualitat del Sistema Universitari a Catalunya, con el objetivo esencial de promover la mejora de la calidad en el sistema universitario catalán.

Uno de los objetivos prioritarios de la ordenación del sistema universitario en Cataluña es la consecución de la máxima calidad en la prestación de los servicios que esta ofrece a la sociedad. Para lograrlo, los estamentos responsables de gestionar y gobernar las universidades catalanas deben desarrollar los instrumentos necesarios para evaluar la calidad docente e investigadora; para implementar programas de mejora y para dar cuenta, en último término, de los recursos dispuestos y el esfuerzo inversor a cargo de los presupuestos públicos ${ }^{34}$.

De la misma manera que lo señalado por los documentos provistos por la propia agencia, dentro de aquellos aspectos más generales que motivaron su creación están el establecimiento de nuevos modelos de financiamiento universitario -los que consideraban el logro de objetivos de calidad-; las experiencias recientes desarrolladas en Cataluña en materia de calidad universitaria; y las nuevas tendencias europeas e internacionales en el tema ${ }^{35}$.

La creación de la Agència per a la Qualitat del Sistema Universitari a Catalunya está en la línea propuesta por el Comité Económico y Social de la Unión Europea, que como se ha señalado anteriormente, recomienda la creación de sistemas transparentes para avanzar en el uso de técnicas de garantía de calidad, con el fin de dar apoyo a las

33 Ver referencia en pie de página $\mathrm{n}^{\circ} 31$.

34 Estatutos de Constitución del consorcio AQU Cataluña.

35 Ver referencia en pie de página $\mathrm{n}^{\circ} 23$. 
universidades y dar soporte para la cooperación y el intercambio de experiencias mutuas ${ }^{36}$.

La agencia nació como un organismo dotado de personalidad jurídica propia, independiente de la de sus miembros ${ }^{37}$, con plena capacidad para el cumplimiento de sus objetivos y sometida a derecho público. Tenía como objetivo impulsar la calidad y la mejora continua del sistema universitario catalán mediante su principal instrumento: la evaluación institucional de la calidad, la que se articulaba en una metodología previamente establecida. También se le encargaba el análisis de los resultados del sistema universitario y la propuesta de medidas de mejora de los servicios que prestaban las universidades públicas catalanas a la sociedad ${ }^{38}$. En este sentido, y de acuerdo con los documentos revisados, su creación constituyó una clara señal del interés compartido por la Generalitat y las universidades públicas catalanas de emprender de manera conjunta, por medio de un consorcio, las actividades de evaluación y de aseguramiento de la calidad de las universidades catalanas ${ }^{39}$. En palabras de su presidente:

AQU Cataluña va a ser una creación espontánea del sistema universitario catalán, una actuación sincrónica del DURSI ${ }^{40}$ y de las universidades públicas que va a hacer manifiesta la preocupación compartida por la calidad y el afán de trabajar conjuntamente para ponernos al nivel de los países más avanzados ${ }^{41}$.

La agencia nació "(...) como fruto de un pacto, con forma de consorcio entre las universidades y la dirección, y bueno, el Ministerio de Educación acá en Cataluña ${ }^{42 "}$.

Esta figura mixta universidad-administración como motor de la creación de una agencia, que además tiene un ámbito no estatal, no es usual y constituye un nuevo modelo que denominamos, el "modelo catalán"³.

36 Ver referencia en pie de página $\mathrm{n}^{\circ} 15$.

37 Memoria Consorcio AQU Catalunya 1996-2003.

38 Documento Institucional AQU: Historia (1996-2003).

39 Ver referencia en pie de página $\mathrm{n}^{\circ} 31$.

40 Departament d'Universitats Recerca i Societat de la Informació de la Generalitat.

${ }^{41}$ Ver referencia en pie de página $\mathrm{n}^{\circ} 3$.

42 Ver referencia en pie de página $\mathrm{n}^{\circ} 11$.

43 Ver también en Rauret (1999). 
Cabe destacar que lo que se designa como el modelo catalán corresponde a la forma en la que se constituye la agencia responsable del desarrollo de dichas evaluaciones. El modelo catalán de agencia consiste esencialmente en una estructura mixta entre la administración y las universidades, en donde debe hacer manifiesta la colaboración y el diálogo existente entre estas entidades tanto en los órganos de gobierno de la misma como en su estructura operativa interna ${ }^{44}$.

Cabe destacar que en su autodescripción la agencia destaca que se trata de un consorcio formado de manera voluntaria por el DURSI y las universidades públicas catalanas ${ }^{45}$. En este sentido, "no es una decisión impuesta por el gobierno, sino que ya recoge una tradición, de consenso de pacto entre todos los agentes..."46. Esta situación contrasta con la realidad de aquellos países europeos pioneros en materia de evaluación de la calidad, los que constituyen básicamente dos tipos de agencia: las creadas por el gobierno (Francia o el Reino Unido) y aquellas promovidas exclusivamente por las universidades (Holanda $)^{47}$.

Se veía muy interesante esta fórmula, pues no venía ni del Ministerio, bueno, como se dice la Consellería, ni tampoco de las propias universidades ${ }^{48}$.

La agencia nace como un organismo sometido al derecho público, dotado, para efectos del cumplimiento de sus objetivos, de personalidad y capacidad jurídica plena (independiente de la de sus miembros), estableciendo su sede en la ciudad de Barcelona ${ }^{49}$. Por lo demás, se puede señalar que la agencia no tiene una orientación al lucro, circunstancia en la que, su presupuesto independiente es financiado, en lo efectivo, por la Generalitat de Catalunya, además de la adquisición de fondos por su participación en proyectos

\footnotetext{
${ }^{44}$ Ver referencia en pie de página $n^{0} 3$.

45 AQU Catalunya proceso de evaluación de la calidad del sistema universitario a Cataluña Informe 2002.

46 Ver referencia en pie de página $n^{\circ} 11$.

47 Ver referencia en pie de página $n^{\circ} 3$.

48 Ver pie de página $n^{\circ} 11$.

49 Ver pie de página $n^{\circ} 3$.
} 
concursables, o por la prestación de servicios a algún organismo que lo requiriera ${ }^{50}$.

Una figura mixta, intermedia (...) que jugaba un papel así de equilibrio, permitía abordar estas temáticas de rendimiento, del sistema y (...) de justificación de los recursos desde una perspectiva más neutral. (...) Un instrumento como la agencia, con la forma jurídica del consorcio, pues daba al sistema mucha agilidad (...) agilidad de gestión ${ }^{51}$.

Es así como en el periodo que media entre su creación (1996) y el 2001, la agencia junto con ordenar y formalizar su funcionamiento interno supervisa el desarrollo del PNECU para las universidades catalanas (1996-2000).

El 2002 se caracteriza por la consolidación plena de la organización, por el esfuerzo en la innovación metodológica y por el establecimiento de las bases para la proyección futura. Se estima importante entonces trazar las metas que tanto la agencia como el DURSI y las universidades deseaban conseguir en materia de calidad, elaborando un plan de actuación que establecería las principales actuaciones que deberían llevarse a cabo durante un periodo determinado (2001-2007). En la elaboración de este plan se procura integrar tanto los objetivos de las entidades que la conforman como de la misma agencia, la que paulatinamente comienza a proyectar su quehacer a estándares que superan el de la sola comunidad autónoma y el Estado español, y ponen su atención en el contexto europeo ${ }^{52}$.

No obstante lo anterior, el Plan de Actuación aprobado el 2000 se pone en marcha en 2001 en medio de un entorno bastante intranquilo para el sistema universitario catalán. Las polémicas derivadas en España de la creación de un nuevo marco legal en materia universitaria, que entre otras cosas creaba una agencia de calidad a escala estatal (cuyas atribuciones parecían incidir en las actividades de la agencia catalana), marcaron el desarrollo del quehacer de las universidades y repercutieron en las actividades de la agencia. A pesar

\footnotetext{
50 Ver referencia en pie de página $\mathrm{n}^{\circ} 12$.

51 Ver referencia en pie de página $\mathrm{n}^{\circ} 11$.

52 Ver referencia en pie de página $n^{\circ} 3$.
} 
del convulsionado ambiente existente, la agencia desarrolló, tanto el 2001 como en 2002, el programa según el plan de actividades establecido para el periodo 2001-200753.

El 2002 marca un nuevo hito en la actividad de la agencia, pues ese año se empieza a gestar la Ley de Universidades de Cataluña (LUC), la que será aprobada el año siguiente. Dentro de los principales elementos que contendrá esa ley se encuentra la reformulación de la agencia existente, en una nueva entidad dotada de mayores atribuciones. Es así como el 2003, mediante la promulgación el 19 de febrero de 2003 de la Ley de Universidades de Cataluña (LUC: Llei 1/2003, de 19 de febrer, d'universitats de Catalunya; DOGC núm. 3826, de 20 de febrer de 2003) se establece también la transformación de la agencia originalmente articulada en un consorcio, en una nueva entidad que, de acuerdo con lo expresado en su preámbulo, se constituirá en el principal instrumento para la promoción y la evaluación de la calidad. La nueva agencia ${ }^{54}$, (AQU Cataluña) tendrá más atribuciones que el antiguo consorcio y se constituirá en una entidad de derecho público, sometida al derecho privado.

Este cambio se introduce para afrontar las nuevas responsabilidades que se derivan de la LOU, proveyendo de esta forma las adecuadas garantías de independencia, profesionalidad y libertad de obrar que caracterizan a las principales agencias europeas.

\section{AQU Cataluña como agencia}

Aun siendo distinta, la nueva agencia (en adelante AQU) es la entidad que da continuidad a la iniciativa desarrollada en un primer momento por el antiguo consorcio, en el sentido de que proyecta su accionar en el tiempo, ampliando las posibilidades de acción de esta. Es así como AQU Catalunya declara que afronta esta nueva etapa con el mismo espíritu emprendedor del consorcio, manteniendo su principios de actuación basados en la ética, la transparencia y la independencia en los resultados de sus actuaciones, como también su voluntad de

\footnotetext{
53 Ver referencia en pie de página $\mathrm{n}^{\circ} 3$.

54 Agència per a la Qualitat del Sistema Universitari "de” Catalunya, por contraposición a la antigua agencia denominada Agència per a la Qualitat del Sistema Universitari "ạ" Catalunya.
} 
innovación; el deseo de desarrollar sistemas de aseguramiento de la calidad adecuados para las universidades catalanas y su vocación europea $^{55}$.

No obstante esta continuidad de inspiración y de intenciones, es necesario destacar que el nacimiento de la nueva agencia estipula de manera explícita el cierre de la anterior, a partir de la disposición derogatoria contenida en los propios estatutos de $\mathrm{AQU}^{56}$.

El paso del antiguo consorcio a la creación de AQU Cataluña va a suponer el cierre de una entidad y la apertura de una nueva, la que tendrá que asumir las funciones de su predecesora y, además, nuevas atribuciones, competencias y estructura. También deberán adaptarse los órganos de gobierno existentes y crear otros nuevos de acuerdo con el marco establecido ${ }^{57}$.

Es así como podemos señalar una continuidad de inspiración, pero una discontinuidad en los aspectos formales que la constituyen.

A tenor de lo que señalan los documentos de la propia entidad, el cambio fue necesario para afrontar las nuevas responsabilidades que se derivaban de la Ley 6/2001 Orgánica de Universidades (LOU), con las garantías de adecuada independencia, profesionalidad y libertad de obrar que caracterizaban a las principales agencia europeas ${ }^{58}$. Observamos así que una de las principales inspiraciones que orienta el accionar de la LUC en este sentido es que:

(...) desde Europa, y desde el punto de vista legal, el reconocimiento de las agencias, responsables de la evaluación de las titulaciones y de la universidad, deben ser independientes, tanto de las unidades evaluadas como del gobierno. Esa es la razón por la cual el consorcio se sustituye por una agencia, de titularidad pública, pero con funcionamiento independiente ${ }^{59}$.

\footnotetext{
55 Ver referencia en pie de página $n^{\circ} 45$.

56 Estatutos de la Agencia para la Calidad del Sistema Universitario de Cataluña.

57 Memoria de actividades AQU 2003.

58 Ver referencia en pie de página $n^{\circ} 23$.

59 Entrevista jefe técnico AQU.
} 
Es así como la LUC va a modificar sus estatutos organizativos y a ampliar sus funciones hacia la acreditación y la certificación. Este "segundo nacimiento" se produce cuando AQU Cataluña ya acumulaba bastante experiencia en metodologías de evaluación, además de un conocimiento profundo del sistema universitario catalán ${ }^{60}$.

Dentro de los principales aspectos que para efectos de la creación de la nueva agencia implicó la LUC se encuentra primeramente el jurídico. Según la LUC, la agencia pasa de ser un consorcio voluntariamente formado por DURSI y todas las universidades públicas catalanas, a ser un organismo autónomo ${ }^{61}$; vale decir, independiente en su existencia de la voluntad concertada de ambas. Tal como lo expresan los documentos de AQU, esta nueva configuración facilitará la comunicación al más alto nivel por medio de su Consejo de Dirección, pues en este órgano estarán presentes los rectores de las universidades catalanas (todas las públicas y algunas privadas) y los presidentes de los Consejos Sociales de las instituciones públicas, lo que configurará un adecuado marco para la cooperación y la confianza ${ }^{62}$.

Por lo demás, la transformación configura a la nueva agencia en una entidad de derecho público que ajusta su actividad al derecho privado. Esta fórmula representará una apertura del sistema a las universidades privadas de Cataluña y le otorgará a AQU Cataluña personalidad jurídica autónoma, plena capacidad de obrar ${ }^{63}$ y patrimonio propio para el cumplimiento de sus funciones, facilitándole una actuación más independiente de las universidades ${ }^{64}$.

En términos estructurales, se adaptaron los órganos de gobierno existentes (Presidencia, Consejo de Dirección, Dirección) de acuerdo con el nuevo marco establecido. En cuanto al Consejo de Dirección,

\footnotetext{
60 Ver referencia en pie de página $\mathrm{n}^{\circ} 23$.

61 Ver referencia en pie de página $n^{\circ} 45$.

62 AQU Cataluña: proceso de evaluación de la calidad del sistema universitario a Cataluña Informe 2004.

63 Al respecto, cabe destacar en este punto que el consorcio ya contaba con personalidad jurídica propia, independiente de la de sus miembros, con plena capacidad para el cumplimiento de sus objetivos, y con un cierto nivel de flexibilidad que facilitaba su accionar.

64 Guía de evaluación externa de enseñanzas universitarias.
} 
por ejemplo, se estableció una nueva composición para él ${ }^{65}$. De la membrecía del Consejo destaca, además de los contemplados originalmente por el antiguo consorcio, la incorporación permanente del rector de la Universitat Oberta de Catalunya; la incorporación variable y condicionada ${ }^{66}$ de hasta un máximo de tres rectores de universidades privadas; la incorporación de tres destacadas personalidades del ámbito académico; además de los presidentes de las nuevas Comisiones de Evaluación de AQU ${ }^{67} 68$.

Sobre esto último, se puede señalar que para el ejercicio de las nuevas funciones encomendadas la LUC determina que al interior de la agencia se formen tres comisiones de evaluación (de la calidad; del profesorado lector y colaborador; y de la investigación), las que tienen la responsabilidad final de aprobar, de manera independiente, las evaluaciones de los ámbitos respectivos ${ }^{69}$. De esta forma, la agencia se dota "(...) de unos órganos que ya no son ni están ligados, ni a las universidades ni al gobierno" $"$.

A partir de los cambios jurídicos y estructurales antes detallados, es como AQU Catalunya asume las nuevas funciones que le encomienda la LUC, estructurando en sus órganos ejecutivos y de gobierno $^{71}$.

65 Ver referencia en pie de página $\mathrm{n}^{\circ} 57$.

66 LUC Artículo 142 c) Los rectores, hasta un máximo de tres, de universidades privadas que hayan adoptado una figura jurídica propia de las entidades sin ánimo de lucro, elegidos por el Consejo Interuniversitario de Cataluña, y que se acojan a la programación universitaria de Cataluña.

67 Ver referencia en pie de página ${ }^{\circ} 57$.

68 Ver referencia en pie de página $\mathrm{n}^{\circ} 23$.

69 Ley de Universidades Catalanas (LUC) del 19 de febrero de 2003

70 Ver referencia en pie de página ${ }^{\circ} 59$.

71 Documento Institucional, ¿Qué es AQU Cataluña? 


\section{Cambio en la interacción con los actores intrauniversitarios, a partir de la evolución de consorcio a agencia}

¿De qué manera el paso de la figura de un consorcio a una agencia autónoma afecta la interacción con distintos actores internos de las universidades catalanas? Para responder esta pregunta, cabe considerar que más allá de los cambios internos, la evolución de AQU Cataluña de un consorcio a una agencia autónoma en propiedad no es inocua en términos de los efectos que tiene en la relación con los actores que al interior de las universidades públicas ofician como interlocutores al momento de implementar las políticas de promoción y aseguramiento de la calidad.

No obstante lo anterior, es posible señalar que el eventual cambio en la percepción hacia AQU y consecuentemente a la forma en que se interactúa con ella, no es la misma en los distintos actores que representan a las diversas universidades frente a AQU. De alguna forma esto es entendible a partir de la presunción de Navarro Yánez (2002), de que la forma en la cual se desarrollan los procesos propios de las políticas públicas (en este caso la implementación de las iniciativas de promoción y aseguramiento de la calidad de las universidades) dependerá de los distintos contextos institucionales, provistos en este caso por las distintas organizaciones universitarias, y de la forma en la cual los distintos actores constituidos en su interior los procesen.

Sobre el particular, el apartado siguiente nos remite a la opinión y a la percepción del cambio en la naturaleza de AQU Cataluña por importantes actores internos a las universidades en materia de calidad, en lo efectivo, vicerrectores responsables de esta materia, de cuatro universidades públicas catalanas, entrevistados entre el 2006 y 2007. Para efectos de anonimatización, en la cita se señala como VRQ, rematando en el nombre de una letra griega para distinguir cuando corresponda a una universidad u otra. Así, por ejemplo, si en la cita sale señalado VRQ Alfa, nos remite a la opinión y percepción del vicerrector en temas de calidad de la universidad a la que, para efectos de la señalada anonimatización, se le ha asignado la denominación de fantasía Alfa (o Beta, Gama, Delta, según sea el caso). 


\subsection{U- Alfa}

En términos generales, es posible afirmar que en la manera en la que el VRQ-Alfa se aproxima a AQU hay un componente crítico notable, derivado del cambio histórico en que las instituciones han entendido su relación con AQU. En este sentido, el VRQ-Alfa ilustra esta situación de cambio de perfil institucional y jurídico de AQU, al señalar que originalmente los evaluadores y evaluados estaban en un mismo lado de la cancha y que posteriormente hay una suerte de escisión de esa comunidad evaluativa.

Si bien las universidades continúan colaborando con las iniciativas respectivas, lo hacen de una manera distinta, pues ya no forman parte de la institucionalidad AQU, salvo de manera muy indirecta. Según los entrevistados, lo anterior deriva de una exigencia de la LOU, que determina que reguladores y regulados ya no se encuentren en un mismo cuerpo ${ }^{72}$.

En términos generales, el trato de AQU con la U-Alfa se percibe, por parte de los entrevistados, como igual al de la agencia con el resto de las universidades, pues, como lo percibe el VRQ-Alfa, AQU se ha blindado y ensimismado (vuelto autista) a partir de una serie de críticas realizadas y presiones ejercidas por otras universidades, en torno al desarrollo de su función evaluativa, principalmente de la docencia. En palabras del VRQ-Alfa el blindaje que ha creado AQU en torno a ella es lo que le impide que se pueda crecer en conjunto manteniendo los roles correspondientes (evaluador y evaluado).

En este sentido, la U-Alfa manifiesta cierto nivel de desazón y desconcierto, además de sufrir cierta sensación de incomprensión por parte de AQU, pues como señala el entrevistado, la tensión que existe entre ambas entidades no es la misma que la que hay con el resto de las universidades. En opinión de la autoridad de la U-Alfa, las otras universidades entran en tensión con la agencia principalmente porque sus profesores no pasan las correspondientes evaluaciones,

72 Conforme con lo señalado en la revisión de los boletines de AQU, como asimismo conforme a lo que informa el propio jefe de la unidad técnica de la agencia, además de una compulsión legal, a este nuevo reformateo de lo que es la agencia, sigue una tendencia a nivel europeo en materia de la constitución de las agencias públicas implicadas en temas de la calidad. 
lo que no es el caso de la U-Alfa. De manera distinta al conflicto de AQU con la U-Alfa, la autoridad de esta última la explica por la forma en que la agencia interactúa con ella en la aplicación de sus métodos evaluativos. En opinión del VRQ-Alfa, esta interacción se da en la actualidad de manera más autoritaria, más impositiva, más autista, menos participativa, menos flexible, más ingenieril y menos sensible a la realidad organizacional de la institución que lo que se daba en un primer momento. En este sentido, es un conflicto que tiene que ver con el cambio de tono de las relaciones entre AQU y las instituciones, en la medida en que se burocratiza y profesionaliza como cuerpo.

Así, si bien el VRQ-Alfa entiende que la evolución natural hacia un mayor nivel de profesionalización no necesariamente debe ser democrático, sí reconoce que el aprendizaje debe ser conjunto. "Tampoco se trata de ser democrático..." (VRQ-Alfa), pero sí que deje ir a las universidades por su tranco "(...) que ya somos mayores..."(VRQ-Alfa).

A pesar de la crítica a su nueva actitud, lo que explicaría en parte la caída en la motivación inicial que había para participar en los procesos, el VRQ-Alfa es capaz de reconocer la función evaluativa de AQU, aunque siempre con reparos. En lo específico, el VRQ-Alfa manifiesta su disconformidad con aquellas acciones maximalistas en materia de evaluación que hegemonizan el proceso.

Al final quieren empezar a querer evaluarlo todo en unos planes muy maximalistas. Donde el resto del profesorado simplemente no puede seguirlos y lo rechaza. ¿Qué estamos haciendo aquí? Modelos, procesos, pero no sirven por maximalistas, excesivamente tecnificados (VRQ-Alfa).

La idea del VRQ-Alfa es que, en los procesos de evaluación no ha de perderse la visión estratégica y de sentido común del asunto, que muchas veces parece encontrarse amenazada por la esencialización de los procesos evaluativos realizados por aquello que él llama "el club de la calidad".

Al final se va a convertir esto como en un club de gente muy sofisticada en procesos donde ellos se acreditan unos a otros y vamos 
a entrar aquí en unos bucles de locura donde todo es burocracia y burocracia (VRQ-Alfa).

En este sentido, el VRQ-Alfa desdeña además aquellas situaciones que identifica como el bucle autorreferente de la calidad, en el cual la propia agencia abocada a promover y evaluar la calidad es objeto de evaluaciones de su calidad. Esto, en su opinión, impone un nivel de rigidez que detona una serie de inseguridades en la agencia, lo que redunda en que esta se ensimisma y en que su relación con las universidades, en especial con la U-Alfa, se tensione.

Yo esto lo discutí mucho con la agencia de calidad. La agencia de calidad [...] tenía una posición excesivamente talibán con temas de etiqueta, de conceptos, su ISO ha sido un elemento más de burocratización de la agencia, más que de mejora de gestión de la agencia [...] un conjunto de instrumentos, que utilizados [...] en la administración pública y especialmente en una administración pública tan especial como es la docente pueden generar pequeños monstruos conceptuales y pequeños monstruos a nivel operativo. Nosotros hemos intentando escapar de esto y ser muy contingentes. [...] Claro que tenemos cosas que aprender, pero nos hemos dotado de nuestros propios instrumentos (VRQ-Alfa).

\subsection{U-Beta}

Para el segundo caso, podemos decir que la forma en la cual el VRQBeta percibe su interacción con AQU es, en términos generales, de alineamiento y colaboración, no obstante debe destacarse que este alineamiento no ignora el cambio de condiciones que ha sufrido la relación entre AQU y las universidades catalanas.

(...) el AQU era, digamos, un consorcio entre las universidades públicas y el DURSI. Digamos que el AQU trabajaba para las universidades, de acuerdo. Pero eso cambió, y pasó a ser una agencia de evaluación institucional controlada solo por DURSI, con lo cual las universidades pasamos a ser objetos de inspección, y lo digo así, crudamente (VRQ-Beta).

(...) eso cambió radicalmente la participación y todo, si antes tú podías pedir información para la planificación, ahora aquí tú eres, te sentías como que eras objeto de la inspección y 
eso se vive... y muchas cosas, se creó mucho más distancia (VRQ-Beta).

(...) digamos que el tono fue igualmente amigable. Pero en cuanto a las funciones realmente que teníamos ahí, y bueno el cambio fue para los rectores. Los rectores pues pasaron de proponer a quejarse. Porque claro que vas a una reunión en la cual pues solo puedes decir... ¡Oye no estoy de acuerdo con esto!... no..., o sea no había otra respuesta posible. En cambio la otra era, qué vamos a hacer, pongámonos de acuerdo en qué hacemos, lo vemos, y yo creo que habría que hacer esto, yo creo que habría que hacer lo otro. En este otro caso AQU hace lo que le da la gana, porque es independiente. Entonces viene la queja (VRQ-Beta).

A pesar de lo anterior, subyace una disposición colaborativa por parte de la VRQ-Beta hacia las actividades promovidas por AQU, no obstante esta se encuentra lejos de ser simple y espontánea, pues en dicha aproximación, y al igual que en el caso de la U Alfa-aunque por motivos distintos-, también se manifiestan críticas a la actual relación de AQU con la U-Beta; así visto podría hablarse de una mixtura donde además de una disposición a la cooperación, podríamos decir basal, se desarrolla una instancia de tensión con AQU, o a lo menos de queja frente a ella.

\subsection{U-Gama}

De manera un tanto distinta de los casos anteriores, la forma en la cual la VRQ-Gama se vincula con AQU puede ser caracterizada en términos generales como la de operar como un enlace político con el quehacer cotidiano de AQU y, en lo que respecta a la evaluación de la calidad de las titulaciones en específico, la sitúa en:

(...) un nivel político, a partir de la reunión que hay de todos los vicerrectores de calidad de las universidades catalanas con la dirección de $\mathrm{AQU}$, que se hace regularmente, y que es para tratar temas de calidad, y otros temas que tienen que ver con AQU (VRQ-Gama).

(...) AQU decidía cada año qué tipo de titulación se tenía que evaluar. De las propuestas que hacía AQU, la universidad veía si tenía alguna titulación en situación de ser evaluada, o sea, 
que habían terminado una promoción entera de estudiantes, y por tanto ya era susceptible (VRQ-Gama).

Como representante político de rectoría para temas de la calidad en la educación, por parte de la VRQ-Gama, existe un interés manifiesto de dotar de visibilidad externa a los procesos desarrollados, lo que se encuentra muy alineado con el discurso de AQU en la materia, y que se manifiesta en una franca disposición a la colaboración.

(...) nuestra universidad siempre ha tenido como objetivo fundamental garantizar la calidad, no solo desde dentro, sino también a través de la visión desde afuera, y en este sentido el rector anterior y el actual son muy, digamos, propensos a buscar referentes externos para ser observados, en este sentido la universidad tenía claro que esto se tenía que realizar... (VRQ-Gama).

En este sentido, no resulta difícil caracterizar la forma en que la VRQ-Gama orienta su interacción con AQU como una instancia de colaboración incondicional, en cuyo marco pareciera incluso que no hace diferencia entre el momento del consorcio y el de la nueva agencia, en el sentido de que ha hecho suya parte importante de los preceptos que subyacen en el discurso de AQU en materia de calidad.

\subsection{U-Delta}

En términos generales también es posible afirmar que desde un primer momento la VRQ-Delta se ha manifestado colaboradora del accionar del AQU en materia de aseguramiento de la calidad y de las distintas iniciativas que la agencia ha desarrollado al respecto en el tiempo.

(...) esta universidad fue pionera, porque digamos que fue la primera... yo no puedo recordarlo porque yo no estaba en este ámbito, pero incluso diría que en algún momento se creó un responsable del tema calidad a nivel de vicerrector, que después ha sido, digamos ha querido integrar en otros vicerrectorados, etc. (VRQ-Delta).

No obstante lo anterior, y a pesar de la cercanía inicial, se manifiesta una clara aceptación y comprensión de que AQU haya dejado de ser un consorcio y se haya transformado en una entidad 
independiente de las universidades, circunstancia desde la cual es posible afirmar que la actitud de colaboración se proyectó más allá de la situación contingente que determinaba el vínculo de la U-Delta con el consorcio en torno al cual se constituía AQU, e incluso una vez "independizada" de las universidades la U-Delta, a través de la VRQ-Delta, compromete colaboración.

Luego el AQU ya pasó a una siguiente fase, en la que ya no era consorcio. Las universidades, yo creo que por la forma natural de perder una posibilidad de influencia, pues de entrada mostraron cierto recelo a esto. Pero yo creo que esto, es decir una agencia de evaluación, por definición tiene que ser independiente... (VRQ-Delta).

(...) creo que es como tiene que funcionar, o sea que, digamos que hay comunicación en el sentido de que tenemos reuniones muchas veces, pero yo creo que va quedando claro que la agencia tiene su papel y las universidades tenemos otra, o sea la división de funciones yo creo que se va consolidando, y yo creo que esto es bueno (VRQ-Delta).

De manera distinta al caso de la U-Gama, la Universidad Delta no es ciega al cambio del consorcio a la agencia, lo que sucede es que tiene contemplada la normalidad de este proceso, circunstancia en la cual no parece sorprenderle que esto haya sucedido. En este sentido, se percibe y se nota el cambio, sin embargo esto no afecta la dinámica global de colaboración de la U-Delta y AQU.

\section{Conclusión}

Respecto de la constitución inicial de la Agencia en torno a la figura de un consorcio, cabe destacar que inicialmente cobra relevancia describir la relación de la recién creada entidad con el PNECU, en el sentido de que la síntesis entre la creación de esta y el convenio suscrito por la Generalitat con el Ministerio de Educación y Ciencia parece ser una solución momentánea a la tensión que generó, en materia de competencias autonómicas, la iniciativa del Plan Nacional de Evaluación de la Calidad Universitaria. 
Esta es la solución que se va a encontrar para resolver el problema inicial de la no implicación de las comunidades autónomas en la evaluación institucional de la calidad ${ }^{73}$.

Es así como, vislumbrada la necesidad de una agencia, 1996 nos presenta la creación -a lo menos nominal- de un actor propio en materia de calidad en la educación superior por parte de la Generalitat, ante aquello que pudiese ser identificado como una inquietante iniciativa hegemónica por parte del gobierno central de Madrid.

Da la impresión de que el rápido acuerdo que surge entre las universidades y la Generalitat, para efectos de la creación de una nueva entidad concertada (lo que destaca como particular de aquello que se ha dado en llamar el modelo catalán), más que a algo espontáneo, obedece a la necesidad de una reacción política, que se presenta como urgente ante las posibles injerencias que las iniciativas en materia de calidad universitaria desarrolladas por el gobierno central pudieran tener sobre un espacio en el cual las competencias autonómicas, en términos de evaluación de la calidad, carecían de un agente responsable claro, y no existían más interlocutores válidos que los tradicionales $^{74}$. Estimados el gobierno central y el autonómico como actores, la dinámica interactiva de este último respecto del primero puede ser calificada como de apresurada defensa de su ámbito de competencia.

Esta situación permite explicar el rápido acuerdo al que se llega en torno a una materia que en otras latitudes resulta tan complicada de acordar entre gobiernos y universidades, y que parece la matriz básica al interior de la que se desarrolla el, así llamado, modelo catalán de agencia ${ }^{75}$, que más que fruto de una reacción espontánea parece hablarnos de una urgida y exigida colaboración frente a la contingencia.

Por medio del acto inicial se crea la agencia como consorcio, no obstante el año de la creación y el siguiente constituyen una suerte

73 Ver referencia en pie de página $n^{\circ} 12$.

74 Ver referencia en pie de página $n^{\circ} 27$.

75 Ibíd. 
de acompasada espera para darle cuerpo institucional a una entidad que, más allá de lo formal, parece existir solo nominalmente. Es así por ejemplo, como durante 1997 el primer director de la agencia opera casi como un regente provisorio a la espera de la asunción en el cargo de una persona con experiencia en el tema específico y con un mayor horizonte de proyección en el tiempo. Esta persona finalmente se encarna en la segunda directora de la agencia, quien ayuda a estructurar el trabajo por desarrollar, incluyendo a expertos en materia de calidad universitaria, de las propias universidades. No obstante fue el énfasis técnico impreso por la nueva dirección lo que determinó el cambio más importante en su momento y que resultó en la evolución del consorcio a una nueva agencia autónoma, posibilitado por las temáticas puestas en evidencia por la LOU (2001) en materia de calidad en la educación y las posibilidades institucionales que brindaba la LUC (2003).

Es solo a partir de estas coyunturas históricas y legales que AQU Cataluña se transforma en una realidad compuesta, que combina elementos de un actor colectivo, con uno de características corporativas (organización). En correspondencia a lo señalado por Scharpf (1997), esta disposición es utilizada para efectos de sintetizar y optimizar "...la rendición de cuentas a los miembros de un actor colectivo y la efectividad superior de un actor corporativo" (p. 57).

Es así como se configura una estructura más compleja, en la cual la asociación entre la Generalitat (por medio del DURSI) y de las universidades catalanas, legitima y controla un cuerpo organizado y dotado de un mayor nivel de autonomía. En la nueva agencia, los órganos de gobierno se estructuran en su parte superior como un cuerpo colegiado dotado de una presidencia, donde los propósitos se formulan de manera conjunta y se deciden, por lo menos, los aspectos más trascendentes, por medio de votaciones.

No obstante lo anterior, la disposición de recursos y la acción de la nueva agencia se organizan por medio de los diversos tipos de órganos que estructuran el resto de la organización y que son conducidos por la dirección de la agencia, la que en este nuevo escenario se vuelve un actor muy poderoso, en tanto es la entidad 
que orquesta, en un esfuerzo técnico de realización, las directrices que se componen de manera asociada en el Consejo de Dirección, la Presidencia y la Comisión Permanente, plasmándolas en el accionar cotidiano de sus otros órganos internos, como la Comisión de Evaluación de la Calidad. El hecho de que, además, quien oficia como directora ejerza la presidencia de esa comisión (además de la presidencia de diversos comités consultivos) ${ }^{76}$ nos habla de un riesgo asumido conscientemente por el conjunto de órganos de gobierno, en pro de mantener la coordinación y el control sobre aquello que identificamos como el core businnes ${ }^{77}$ de AQU Cataluña: la evaluación institucional de la calidad de las titulaciones.

Al respecto, resulta ilustrativo señalar que según Scharpf (1997), una organización burocrática (como aquella que se perfecciona con la figura de la nueva agencia) en sí misma es, por una parte, el mayor recurso activo de la asociación, pero por la otra, es el más poderoso instrumento por medio del cual los líderes son, potencialmente, capaces de controlar y explotar a la corporación y sus miembros.

Sobre el particular, y desde la perspectiva del accionar de la Comisión de Evaluación de la Calidad y de las iniciativas que por medio de esta se conducen al interior de las distintas universidades, es que la relación entre estas últimas y AQU varía, como también varía la forma en que es percibida la relación con la agencia por parte de aquellas, ejemplificadas en la reacción de sus máximos representantes institucionales. De alguna forma, parece que esta instancia es el espacio más sensible donde la mayoría de los entrevistados acusa el cambio de tono en la relación entre AQU y su institución y no pocos la resienten.

Respecto de esta dinámica interactiva, la propia deriva legal e institucional en la cual se inserta AQU determina que en el tiempo mute sus principales características: primero como un consorcio, al parecer constituido de manera apresurada para resguardar ciertas prerrogativas de la comunidad autónoma que, en tanto no terminadas de asumir, amenazaban un importante ámbito de autonomía de la

\footnotetext{
76 Ver referencia en pie de página $n^{\circ} 57$.

77 Ibíd.
} 
comunidad catalana en materia de educación universitaria. En el marco de esta premura es que se ensaya un modelo cooperativo concertado (consorcio), de manera tal de privilegiar los acuerdos y la acción conjunta de la comunidad autónoma ante la eventual amenaza de la hegemonía del nivel central del Estado español, en torno a la evaluación de la calidad de las universidades. La evolución a una agencia dotada de mayores niveles de autonomía determina además del cese del tono participativo hacia un claro cambio de sintonía en su relación con las universidades que anteriormente participaban del consorcio original, pudiendo aludirse, en algunos casos, a un claro distanciamiento, el que si bien parece ser explicable para muchas de ellas, no deja de ser ingrato en términos del cambio en la dinámica interactiva con la entidad de la cual ya no se es parte. Desde la perspectiva del ACI, podemos decir que, junto con la mutación de consorcio a agencia, han variado las especificaciones del setting institucional, dejando las universidades de ser partícipes de un proyecto evaluativo y pasando a ser objeto de un sistema de evaluación. La pérdida del tono colaborativo de las relaciones entre AQU y las universidades de alguna manera afecta el desarrollo de los programas de evaluación de la calidad de las titulaciones, en tanto estos comienzan a ser supervisados por una entidad externa, cada vez más ajena. En este marco, la discursividad inicial que nos remitía a la colaboración y a la confianza de un pretendido modelo local, pasa a ser reemplazada por una serie de aprehensiones por parte de los máximos representantes en materia de los programas de calidad (VRQs) de las diversas universidades revisadas.

La forma en que este cambio es procesado por las universidades varía desde cierto nivel de molestia (U-Alfa y U-Beta) hasta la resignada asunción de una realidad prevista con antelación (U-Delta), pasando por la obsecuente disposición a colaborar por parte de una institución intensamente identificada con las políticas de calidad (U-Gama). Lo que se quiere señalar con esta reflexión es que el cambio de los atributos y capacidades institucionales de la agencia original, asociado a la deriva de su contexto institucional y legal, implicó necesariamente un cambio en la forma del sistema de interacción general que sostenía la agencia con el conjunto de universidades, así como del sistema específico de interacción que desarrollaba con cada una de ellas. 
Evidentemente esto puede ser leído desde la lógica de la tecnificación y profesionalización del trabajo de la agencia según estándares de orden europeo, pero para efectos de un análisis concreto de las condiciones en que se desarrollarán las futuras interacciones con las universidades, no deja de ser un desilusionante cambio en las condiciones iniciales de interacción, que originalmente promovían la colaboración entre las distintas universidades con el consorcio original, a un esquema en donde la dinámica reconoce a la agencia como actor central, la que impone una dinámica más instructiva y evaluativa a instituciones universitarias.

De alguna forma la dinámica que se consolida gira en torno a un organismo que, para efectos de la implementación de los programas de evaluación de la calidad de las titulaciones, es percibido más controlador que promotor, algo que en los momentos preliminares a la gestación del consorcio era temido como un peligro, en tanto ni en el mundo universitario ni en la administración pública existía la tradición de evaluar la calidad institucional ${ }^{78}$. Según nuestro esquema de análisis, evidentemente la concreción de ese temor original incide en la forma en la cual los programas de evaluación de la calidad de las titulaciones se implementan al interior de las distintas universidades observadas.

\section{Referencias}

Enders, J., Jeliazkova, M., \& Maassen, P. (2003, September). Higher education policy formulation and implementation: A framework for case analysis. In CHER 16th annual conference "Reform and Change in Higher Education: Renewed expectations and improved performance".

Fernández Lamarra, N. (2007). Educación superior y calidad en América Latina y Argentina. Los procesos de evaluación y acreditación. Buenos Aires: IESALC/UNESCO-EDUNTREF.

Geoffroy, E. (2013). Estudio del Sistema de Aseguramiento de la Calidad de la Educación Superior. Un análisis politológico de formulación de política pública. Revista Enfoques: Ciencia Política y Administración Pública, 11(19)139-166

\footnotetext{
$\overline{{ }_{78}}$ Ver referencia en pie de página $\mathrm{n}^{\circ} 12$.
} 
Harvey, L., \& Green, D. (1993). Defining quality. Assessment \& Evaluation in Higher Education, 18(1), 9-34.

Lemaitre, M. J. (2002). Quality as politics. Quality in Higher Education, 8(1), 29-37.

López Pereira, J. E. (2004). Competencia de las comunidades autónomas en relación con la gestión universitaria. En Tobarra, P. (2004). Planificación estratégica y mejora de la calidad en las universidades (203-214). Consejería de Educación y Cultura, Dirección General de Universidades, Murcia.

Perellón, J. F. (2005). Nuevas tendencias en políticas de garantía de calidad en la educación superior. Papers, 76, 47-65.

Poole, B. (2005). Quality problems. English Today, 21(4), 32-35.

Rauret, G. (1999). El modelo Catalán de Agencia para la Garantía de la Calidad Universitaria. Cuadernos IRC, 3, 39-44.

Scharpf, F. W. (1989). Decision rules, decision styles and policy choices. Journal of Theoretical Politics, 1(2), 149-176.

Scharpf, F. W. (1997). Games real actors play: Actor-centered institutionalism in policy research (Vol. 1997). Boulder, CO: Westview Press.

Scharpf, F.W. (2000 a). Interaktionsformen. Akteurzentrierter Institutionalismus in der Politikforschung, Leske + Budrich, Opladen.

Scharpf, F.W. (2000 b). Institutions in comparative policy research. Comparative Political Studies, 33(6-7), 762-790.

Navarro Yánez, C. J. (2002, octubre). La sociedad política como agenda de investigación: delimitación conceptual y marcos analíticos. En VII Congreso Internacional del CLAD sobre la Reforma del Estado y de la Administración, Lisboa, Portugal (pp.8-11).

Zurbriggen, C. (2006). El institucionalismo centrado en los actores: una perspectiva analítica en el estudio de las políticas públicas. Revista de Ciencia Política, 26(1), 67-83.

Recibido: 24/02/2014

Aceptado: 29/04/2014 